\title{
An independence of mechanism in the origins of reading and classification-related semantic distance effects
}

\author{
A. J. SANFORD, S. GARROD, and J. M. BOYLE \\ University of Glasgow, Scotland, United Kingdom
}

\begin{abstract}
Some members of prespecified classes are accepted as members more rapidly than others. This has normally been ascribed to processes operating at a stage where the members are evaluated with respect to the category. An alternative locus could be at an "encoding" stage. It is shown with two experiments that this seems most unlikely, and evidence emerges to support the idea that word-naming and classification tasks show a crucial independence of process. It would seem to be necessary to reevaluate any simplistic notions of processing stages in semantic verification tasks.
\end{abstract}

In a class membership verification task, subjects are required to decide whether target items are members of specified categories. This procedure has been used to draw inferences about how information is stored in memory. Collins and Quillian (1972), for example, suggested that it takes longer to decide that a collie is an animal than to decide it is a dog because the fact that a collie is an animal is not stored directly, but has to be inferred from the fact that a collie is a dog and a dog is an animal. They assume that factverification times could be explained in terms of the number of steps involved in a chain of inferences. An alternative view (Landauer \& Meyer, 1972) is that when a category (e.g., "dog" or "animal") is presented along with a target, then the subject searches through the exemplars specified by the category until the target is found. Searching for collie as a member of a large category (animal) would take longer than finding it in a smaller set (dog). Both of these theories make assumptions about how data are represented in memory and how checking out the information takes place.

At the empirical level, the facts are simply that verification time varies systematically with the relationship between the item and the category. The norms of Battig and Montague (1969) illustrate that, given an instruction to think of examples of a given category, subjects will think of some exemplars more readily than others. Given "bird," for example, "robin" is more readily thought of (normatively) than is "chicken" or "goose." Wilkins (1971) demonstrated that the examples less readily thought of (low conjoint frequency or LCF items) were accepted as members of the class more slowly than readily thought of examples (high conjoint frequency or HCF items). Similar observations have been made by numerous other workers (e.g., Rips,

This research was supported by Grant HR $1823 / 1$ to the first author from the Social Science Research Council and by a SRC studentship to the third author.
Shoben, \& Smith, 1973; Rosch, 1973) and, recently, Smith, Shoben, and, Rips (1974) have demonstrated that the category-size effect can be reversed by systematically varying the frequency with which an exemplar is thought of as being an instance of a given category. It is likely, therefore, that the specification of a thing as a member of a class is not all-or-none, but is a matter of degree, and that verification times result from the "strength" of the specification or from how typical the instance is of the class.

These effects (which will be called conjoint frequency effects-CFEs) can be readily thought of as originating at the comparison stage of processing, that is, the stage at which the semantic properties of category and instance are evaluated against each other. Landauer and Meyer's (1972) category search model, which supposes that the presented instance is checked against a list of category members in memory, can accommodate the effect. If a match is achieved earlier for HCF than for LCF items, then the CFE would result (Rosch, 1973). Collins and Quillian (1972) could accommodate the results by supposing there are more intermediate nodes between LCF items and categories than there are between HCF items and categories. A third view ascribes at least some of the CFE to a check for semantic relatedness between category and instance, prior to checking the nature of the relationship (Smith, Shoben, \& Rips, 1974). These three views are all concerned (in different ways) with how the comparison of category and exemplar takes place.

It is also possible to ascribe the CFE to an earlier stage in the processing chain, namely, the encoding stage. It has been recognized for a long time that context influences the perception of words. For example, Morton (1964) showed that context in the form of an incomplete sentence greatly reduced the tachistoscopic recognition threshold of a word which would complete the sentence. Jacobson (1973) showed that, when two contiguous words are vocalized as they are presented, 
the time to read the second word is reduced if it is an associate of the first word. The latter result was confirmed by Meyer, Schvaneveldt, and Ruddy (1975), who used a much improved design. In general, the speed of reading a word is considered to be improved by the presence of some contextual support ("priming" effect).

Consider a class membership verification task in which the category (e.g., "bird") is prespecified, and then targets (e.g., "robin" or "goose") are presented. It is possible that the HCF exemplar (robin) receives more contextual support from "bird" than does the LCF exemplar (goose), and that it is, therefore, read more rapidly, and passed on to the comparison stage more rapidly. If a sufficient difference in contextual support occurred, it could possibly account for the whole of the observed CFE in the verification task, without attributing any of the CFE to further differential effects at the comparison stage.

Figure 1 makes explicit the idea that there are stages in common between the task of vocalizing a word after reading its category name, and deciding whether a word is a member of a prespecified category. Both entail an encoding stage where contextual support (priming) might have an influence.

This way of looking at the processing chain in the evaluation of simple concepts represents the everyday view that silently reading or "encoding" the words (i.e., being able to name them) precedes the extraction of semantic information. The reading task merely involves verbalizing the word, while the classification task involves accessing long-term memory for relational information.

If the model embodied in Figure 1 were correct, then it would be possible to determine whether the CFE in the classification experiment resulted from differential priming at the encoding stage, simply by comparing the magnitudes of the two effects for a common set of materials. If the effects were the same size, then perhaps the CFE could be attributed to encoding, while, if the CFE were larger than the differential priming effect, then the involvement of some additional agency at the concept comparison stage would be suspected.

In reality, such a simple comparison is not sufficient.

(a)

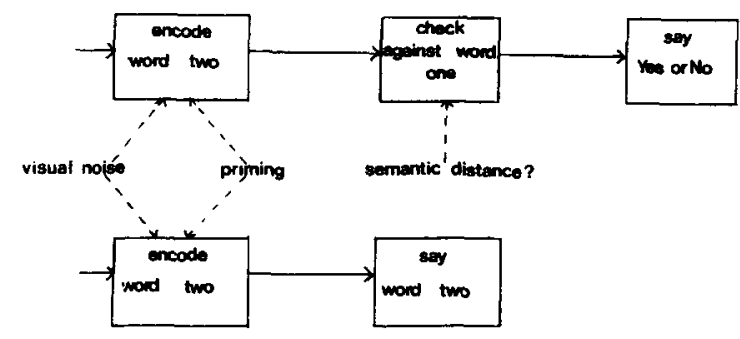

Figure 1. Hypothetical stages in the processes of (a) making a class membership decision, and (b) reading the second of a pair of words out loud. A common stage called "encoding" may be assumed to be an element in both tasks.
Even if classification CFE and differential priming in reading were identical in magnitude, one would still wonder if this were spurious. It is eminently possible that Figure 1 represents incorrect assumptions, and that the contextual priming effects obtained in reading do not influence the "yes" times in the verification task: Verification may operate in a parallel, largely independent manner. This dilemma can be resolved by comparing the influence of a variable known to influence one task to its influence on the other task. Meyer et al. (1975) provide some evidence that mild degradation of the stimuli used in the articulation task not only increases reading times, but also increases the magnitude of the associative priming effect obtained. (This could be thought of as meaning that words which are difficult to read benefit most from context.) If there are differential priming effects in reading for HCF and LCF exemplars, then this differential would be expected to increase if the words being read were degraded slightly. Furthermore, if such priming differentials occur as a stage in the classification task (see Figure 1), then degradation should have the same kind of effect in the classification situation. A number of specific models can be described which make these predictions in an explicit fashion, and one of them will be mentioned in the discussion section of this paper.

For the moment, the argument may be recapitulated as follows: It is suggested that the CFE may result from the different degrees of priming produced in HCF and LCF exemplars by reading a category name. The difference would be reflected in, and indexed by, the difference in reading times for the two exemplar types, with HCF exemplars being read more rapidly. The differential priming effect should be increased by degrading the quality of the words presented. If the magnitude of the differential priming effect is equal to the CFE obtained with the same stimuli in a yes-no classification task, then the CFE may result entirely from such early encoding operations. (This would necessarily involve visual degradation influencing differential priming in reading and the CFE in classification to equal extents.) On the other hand, if the CFE in classification is larger than the differential priming effect, then some other processing stage must be implicated. Finally, in order to invoke the encoding process as a stage in classification at all, it must be influenced by visual degradation, as would be expected with reading.

The idea of using encoding priming as a possible account for the CFE produces predictions relating to a rather different phenomenon. This provides an additional and independent opportunity to test the involvement of differential priming in classification. A requisite of the classification paradigm is that subjects see some cases in which the category name is followed by a nonmember, requiring a "no" response. (This usually occurs on $50 \%$ of the trials.) There is ample evidence that, if the nonmember is related in some way 
to the category, then it is slower to be classed as a nonmember (e.g., Collins \& Quillian, 1972; Schaeffer \& Wallace, 1970). Such a finding cannot be explained in terms of differential priming, since the related item should be primed (facilitated) and the unrelated item should be unaffected by prior exposure to the category. This would mean that, if encoding bias were operating, the related items should be dealt with and rejected more rapidly than the unrelated items, which is the opposite of the effect actually obtained. For this reason, the present authors suppose the related-negative effect to be the result of some other operation, occurring later in the chain of events, such as checking the nature of the connection between the words displayed (Collins \& Quillian, 1972), or the operation of some kind of response bias based on semantic similarity (e.g., Schaeffer \& Wallace, 1970; Smith, Shoben, \& Rips, 1974). However, the fact remains that, on the basis of Figure 1, the associated items should be primed at the encoding stage. The related-negative effect (RT related - RT unrelated) will equal the postencoding effect of relatedness minus the effect of encoding bias. In other words, the observed related-negative effect is less than the true effect resulting from the semantic comparison stage. The effect of visual degradation should increase the encoding-bias effect by slowing down the unrelated items more than the related items, resulting in a net decrease of the observed relatedness effect.

The remainder of the present paper consists of an experimental exploration of the ideas inherent in Figure 1. In the first experiment, the basic proposition that reading a category name will result in faster reading of appropriate HCF than LCF exemplars is examined. Experiment 1 also checks that related nonexemplars are read more rapidly than unrelated nonexemplars. At the same time, the relationship between these effects and visual degradation is examined. The materials were chosen so as to be suitable for Experiment 2, in which the classification paradigm is employed for comparison purposes. Experiment 1 can be thought of as a check that the priming effects, degradation effects, and their interactions hold in the predicted manner for the particular set of materials selected.

\section{EXPERIMENT 1}

\section{Method}

Stimulus materials. For the category-member set, there were 16 conceptual categories, taken from the Battig and Montague (1969) norms. Each category was defined by one word, and was chosen so that an HCF and LCF exemplar could be found, while preserving word frequency and word length across the CF levels. For the nonmember set, for each of the 16 categories, two nonexamples were chosen, so that one word was conceptually related to the category, and the other was not. For the category "fruit," for example, "juice" was selected as a related item, and "major" as an unrelated item. Judgments as to relatedness were made intuitively, and a group of five judges confirmed that the supposed related items were in fact relatively more related than the unrelated items, for all categories used. (There is no ready way of varying relatedness in a more systematic

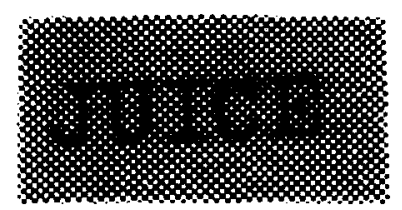

Figure 2. Showing the kind of visual noise used in the two experiments.

manner.) Related and unrelated words were matched for word length and word frequency.

The words were printed on white cards in uppercase letters in Elite type, and were printed in both clear and degraded form. Degradation was achieved by mounting a panel of Letraset "Letratone" over the typewritten word, as shown in Figure 2. Pilot work suggested that this level of difficulty should provide a differential in reading ease sufficient to yield a main effect of degradation, without making it impossible to read each of the words. Stimuli were printed centrally on the cards.

Procedure. Stimuli were presented in a threc-field tachistoscope. On any given trial, the name of a category was presented for $2 \mathrm{sec}$, during which time the subject had to read the word aloud, as rapidly as possible. The target card followed, which could be either a member or a nonmember of the previously presented category. Subjects had to read out the name on the target card as rapidly as possible, as well. A throat microphone coupled to a voice key detected the onset of vocalization of the target. Reaction times (RTs) were measured from the onset of the display of the target card to closure of the voice key.

For a given subject, half of the target words were category members (creating class-member trials), and the remainder were nonmembers (nonmember trials). Half of each trial type were degraded presentations, and the remainder were clear. Thus, if a subject saw a given target in the degraded condition, he did not see it in the clear condition. The words seen in the degraded condition by half of the subjects were seen in the clear condition by the remaining subjects. For any given subject, both the HCF and the LCF exemplars of a given category appeared in the same degradation condition. Similarly, for the nonmember set, both the related and the unrelated items for any given category appeared in the same degradation condition. The design thus enabled all targets to appear in both relevant conditions, without repeating a target exposure to a given subject.

Subjects. The subjects were 12 undergraduate students from the University of Glasgow. They were paid $£ 0.50$ for a single experimental session and were naive as to the aims of the experiment.

\section{Results and Discussion}

Errors. The error rate was low, at $2 \%$, and resulted from coughing or swallowing just prior to making a response. Degradation had no systematic effect on error rate.

Reading times. The means of individual subjects' geometric mean reading times are shown in Figure 3.

Class-member and nonmember trials were analyzed separately. The means for each subject in each condition were calculated, and an analysis of variance was carried out on the data, with subjects treated as a random factor. A similar analysis of variance was also carried out on the mean time for each category, thus treating materials as a random factor and subjects as a fixed effect. This procedure enabled the calculation of the min $F^{\prime}$ statistic (Clark, 1973).

Consider first the class-membership responses: 


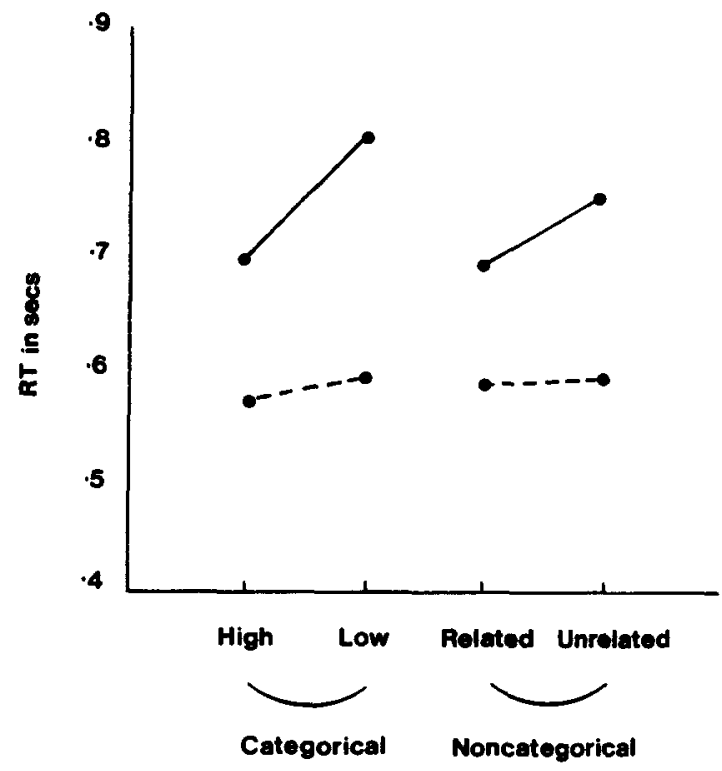

Figure 3. RTs for the reading task. Solid lines indicate measures under noise; broken lines indicate measures without noise. Note the presence of an interaction between the CFE and the relatedness effects and visual noise.

HCF members were read more rapidly than LCF members, by an average of $62 \mathrm{msec}(\mathrm{SE}=19 \mathrm{msec}){ }^{1}$ $\left[\min F^{\prime}(1,26)=5.00, p<.05\right]$. There is also a main effect of degradation, degraded materials being read $176 \mathrm{msec}$ ( $\mathrm{SE}=31 \mathrm{msec}$ ) slower than clear materials $\left[\min F^{\prime}(1,25)=18.6, p<.001\right]$. The interaction of the two, apparent in Figure 3 , is significant both by subjects $[F(1,11)=7.76, p<.025]$ and by materials $[F(1,22)=5.1, p<.05]$, producing a marginally significant $\min F^{\prime}\left[\min F^{\prime}(1,26)=3.08, p<.1\right]$. The data thus show reasonably respectable evidence for an interaction in the expected direction, with LCF items being slowed down more by degradation than were HCF items, by some $100 \mathrm{msec}(\mathrm{SE}=15 \mathrm{msec}$ ).

A similar pattern of results emerged with the nonmember trials. There was a relatedness effect, with related nonmembers being read more rapidly than unrelated nonmembers, by an average of $56 \mathrm{msec}(\mathrm{SE}=8 \mathrm{msec})$, an effect reliable by subjects $[F(1,11)=19.27$, $\mathrm{p}<.005]$, but, unfortunately, not reliable by materials $[F(1,15)=3.85, p<.1]$. Degradation increased reading time by an average of $161 \mathrm{msec}$ ( $S E=23 \mathrm{msec)}$ $\left[\min F^{\prime}(1,26)=18.64, p<.001\right]$. The interaction of Degradation by Relatedness was significant both by subjects $[F(1,11)=5.36, p<.05]$ and by materials $[F(1,15)=5.82, p<.05]$. This yields an unreliable min $F^{\prime}(1,25)$ of 2.79 . Thus, the relatedness effect is larger under degradation, as expected, by some $57 \mathrm{msec}$ (SE for interaction $=12 \mathrm{msec})$. Despite problems of generalizability across the materials domain, the net pattern of results across subjects shows evidence for an interaction between degradation and relatedness. It would take only a few related-unrelated pairs not showing an effect, for the materials effect to fail, while, given a sufficient percentage of pairs showing the effect, it should be maintained by subjects. It should be noted that the present authors are not trying to claim that all related-unrelated pairs that could be generated in the fashion used in this experiment must show a relatedness effect. Rather, it is suggested that, given such an effect reflected in the body of the words as a whole, this will be larger under degraded than under clear conditions, a contention certainly borne out by the data. With this caveat, it is suggested that the nonmember data show an interaction between priming and visual degradation, and, together with the results of the class-member trials, support the general arguments outlined in the introduction.

In summary, HCF items following a class name are read more rapidly than LCF items, and the effect is exaggerated by degradation. Indeed, the effect almost entirely results from the degraded trials. Similarly, items noncategorically related to the previously exposed class name are read more rapidly than unrelated items, although the effect is really only present under degradation. Thus, the results of Experiment 1 provide a baseline against which the encoding-bias explanation of the CFE in classification can be evaluated. Experiment 2 provides data for a comparison of the two tasks.

\section{EXPERIMENT 2}

\section{Method}

Stimulus materials. The materials used were the same as in Experiment 1, that is, the same cards were used.

Procedure. The apparatus used was exactly the same as in Experiment 1. The category name was displayed for $2 \mathrm{sec}$, followed by the target card, which remained visible for 2 sec. Subjects were asked to react as quickly as possible after the onset of the target, and RTs were measured in milliseconds from the target onset. Half of the subjects were instructed to press a key under their dominant forefinger if the target was not a member of the prespecified class, and to press the key under their nondominant forefinger if the target was a member of the class. Finger-response allocations were reversed for the remaining subjects. Subjects received eight practice trials, prior to receiving the main sequences, in which the stimuli were arranged in a different random order for each subject.

For a given subject, half of the target words were category members, and half were not. Furthermore, half of the trials of each type were degraded presentations and half were clear. If a subject saw a word under the clear condition, he did not see it under the degraded condition. Half of the words were seen by half of the subjects under the clear condition and the other half in the degraded condition, the remaining combinations being seen by the other half of the subjects. For any subject, both the HCF and the LCF examples of a given category appeared in the same degradation condition. Similarly, for nonmembers, both the related and the unrelated items for any given category appeared in the same degradation condition. The partitioning of materials employed was the same as that in the previous experiment, to facilitate comparison between the tasks.

Subjects. There were 16 subjects, mostly undergraduates 
from the University of Glasgow, who were paid $£ 0.50$ for a single experimental session; they were naive as to the aims of the experiment.

\section{Results and Discussion}

Errors. The overall error rate was low, at 5.3\%. The breakdown of error types is shown in Table 1 . There was a tendency for more errors to be made in verifying LCF than HCF exemplars; each of the 13 subjects who produced errors on these trials showed this trend $(\mathrm{p}<.01$, sign test). Also, there was a tendency for more errors to be made with related rather than unrelated negatives, with 9 out of the 10 subjects who made errors on the negative trials conforming to this pattern $(p<.011$, sign test). The pattern of results indicates that higher error rates and slower RTs (described below) occurred together; this allows the bypassing of questions of speed-accuracy tradeoff when considering the RT data. Degradation had no reliable effect whatsoever on error rate.

Correct RTs. Figure 4 shows the mean RTs for correct responses, derived from the geometric mean RTs for individual subjects. Consider first the "yes" responses. An analysis of variance carried out on both subjects and materials revealed a strong CFE $\left[\min F^{\prime}(1,23)=13.98, p<.01\right]$. HCF exemplars were classified some $121 \mathrm{msec}(\mathrm{SE}=14 \mathrm{msec})$ faster than were LCF exemplars. Degradation increased RT by $127 \mathrm{msec} \quad(\mathrm{SE}=24 \mathrm{msec}) \quad\left[\min \mathrm{F}^{\prime}(1,28)=18.3\right.$, $\mathrm{p}<.01]$. In contrast to Experiment 1, the interaction is statistically nonsignificant $\left[\min F^{\prime}(1,19)=.12\right]$. The interaction does not approach significance when tested by independent Fs derived from analyses of variance with subjects and materials treated separately [by subjects, $F(1,15)=.14$; by materials, $F(1,15)=.95]$. Furthermore, the interaction mean effect was slightly in the unexpected direction: That is, degradation increased RTs to HCF items by slightly more than it did RTs to LCF items (effect magnitude $=11 \mathrm{msec}, S E=14 \mathrm{msec}$ ). Thus, while the results show strong main effects of CF and degradation, the absence of an interaction stands in marked contrast to the results of the first experiment, and goes against an explanation of the CFE in terms of encoding operations as conceptualized in Figure 1.

A similar analysis carried out on the "no" responses revealed much the same picture. There was a strong effect of relatedness, RTs for related items being longer by some $102 \mathrm{msec} \quad(\mathrm{SE}=15 \mathrm{msec})$ than RTs for

Table 1

Error Rates (as Percentage) in Experiment 2 as a Function of Condition

\begin{tabular}{lrrrcc}
\hline & \multicolumn{2}{c}{ "Yes" Responses } & & \multicolumn{2}{c}{ "No" Responses } \\
\cline { 5 - 6 } \cline { 5 - 6 } & HCF & LCF & & Related & Unrelated \\
\hline Degraded & 2.0 & 12.0 & 7.0 & .7 \\
Clear & .7 & 12.0 & 5.0 & 2.0 \\
Total & 1.5 & 12.0 & 6.0 & 1.5 \\
\hline
\end{tabular}

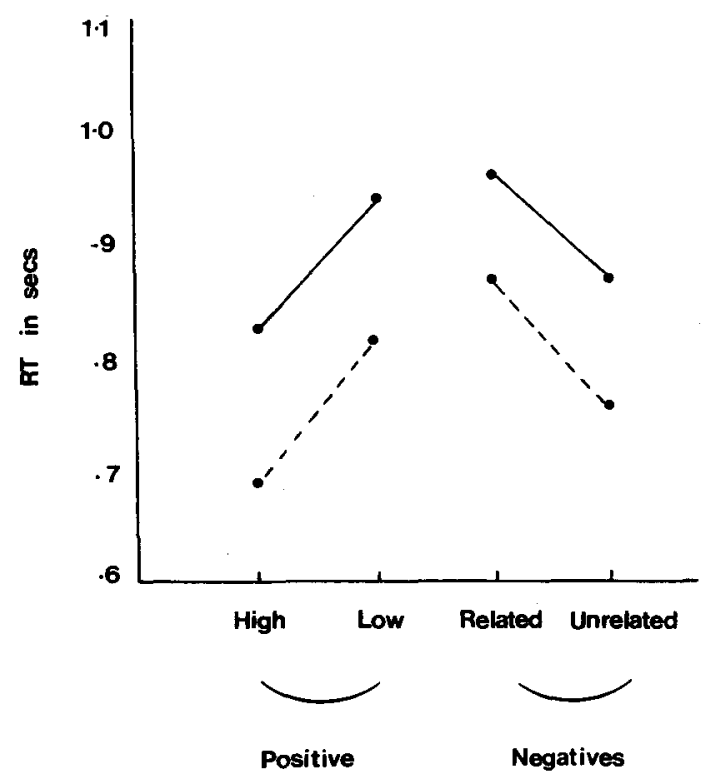

Figure 4. RTs in seconds for the classification task. Solid lines indicate measures under noise; broken lines indicate measures without noise. Note the apparent absence of an interaction between the CFE and visual degradation, and the relatednegative effect on degradation.

unrelated items $\left[\min F^{\prime}(1,29)=29.50, \quad p<.01\right]$. Degradation slowed RTs by $103 \mathrm{msec}(\mathrm{SE}=26 \mathrm{msec})$ [min $\left.F^{\prime}(1,28)=10.47, \quad p<.01\right]$. The interaction of - Relatedness by Degradation was not reliable $\left[\min F^{\prime}(1,25)=.58\right]$. It was similarly unreliable when analyzed by subjects and materials separately [by subjects, $F(1,15)=.81$; by materials, $F(1,15)=2.04]$. The interaction was numerically in the expected direction, but was only a small effect at $20 \mathrm{msec}$ $(\mathrm{SE}=17 \mathrm{msec}$ ).

Since the major difference between the results from the two experiments was in the presence or absence of the interactions, a worthwhile consideration is the power of the analyses employed. From the error variance, it is possible to calculate how large a true interaction would have to have been in order to have been detected in Experiment 2 (Kirk, 1968). Assuming $1-\beta=.90$, the analysis used is capable of detecting an interaction between CF and degradation of $81 \mathrm{msec},{ }^{2}$ which is substantially smaller than the $100-\mathrm{msec}$ effect obtained in Experiment 1. However, an interaction of relatedness and degradation of $82 \mathrm{msec}$ would have been detected, which is more than the $57 \mathrm{msec}$ actually obtained in Experiment 1. It is, perhaps, worth reiterating that the actual magnitudes found in Experiment 2 for these two interactions were $-11 \mathrm{msec}$ and $20 \mathrm{msec}$.

\section{GENERAL DISCUSSION}

At the outset, it was asked whether differential priming at an encoding stage could account for any or all of the CFE. A comparison of Figures 3 and 4 
provides a somewhat complex answer. If a direct comparison had been made between the differential priming effect and the CFE under clear conditions, such a small differential priming effect would have been found that it would have been tempting to conclude that encoding biases were of minor importance in determining the CFE. By contrast, if a direct comparison had been made under the visually degraded condition, it would have been tempting to suppose that encoding biases accounted for the CFE in its entirety. Indeed, with characters of different degrees of legibility, any intermediate result could presumably have been obtained. However, when both degradation conditions are examined together in the present study, what is most striking is that degradation affects the two tasks differently. While it interacts with priming in the reading task, it does not interact with the CFE in classification.

The implication of the present results is that the CFE cannot result from encoding bias totally, if at all. Other experimental work has shown that the CFE in classification can be readily and dramatically increased by various procedures unlikely to operate at the featureextraction stage (if one thinks in the conventional terms of independent successive stages). Requiring a subject to say "no" to category-exemplar pairs and "yes" to category-nonexemplar pairs has such an effect (Sanford \& Seymour, 1974), as does the insertion of "not" into class statements, for example, "A CANARY IS NOT A BIRD-Yes or No?" (Sanford \& Garrod, Note 1). This evidence argues for the involvement of processes other than those conceptualized as "encoding" in terms of Figure 1 above, and, taken together with the present results, indicates that some alternative generator for the CFE should be sought in the comparison process.

The weak conclusion from the present experiments is, therefore, that the CFE cannot be accounted for totally in terms of encoding bias. This applies whether it is assumed that the absence of significant interactions with degenerations reflects a true independence of noise and CFE (and relatedness in the case of negatives), or whether it is assumed the interactions are so slight as to be undetectable by the present design. The question of whether the latter assumption is correct cannot be completely resolved by the present data, but it can be resolved to a certain extent. Morton (1970) has put forward a model which provides an alternative to supposing a complete independence of the operations involved in reading a word and in using its meaning. Morton (e.g., 1970) has suggested that access to information stored in semantic memory is made through the logogen system during reading. The printed word provides features from which is extracted information that is accumulated in the relevant logogen (i.e., the logogen for that word). Degradation of the visual stimulus can be thought of as reducing the rate at which information can be effectively extracted, and hence accumulated at the logogen. A second element of the logogen system is a criterion level for the extracted information at each logogen. When the accumulated information reaches this level, the threshold is passed and the logogen can address later stages in the information processing chain. If the rate of extraction of evidence is lower under degradation, then the time taken to reach the criterion level will be longer. In this way, the model can handle the effects of degradation. It also has no trouble in providing an explanation of the interaction between context and degradation. Context effectively lowers the threshold of the logogens relevant to the context. ${ }^{3}$ The interaction of priming (context) with degradation can be seen to result from Figure 5. The logogen model thus provides a concrete example of the manner in which the "encoding" stage of Figure 1 might work.

Morton's system is complicated by the fact that the same criterion level is not utilized for all tasks. He suggests that the semantic system may be accessed after a lower criterion amount of information has been extracted than would be required to produce a word in the form of a phonological output. Morton (1970) suggests that context (priming) effectively reduces the

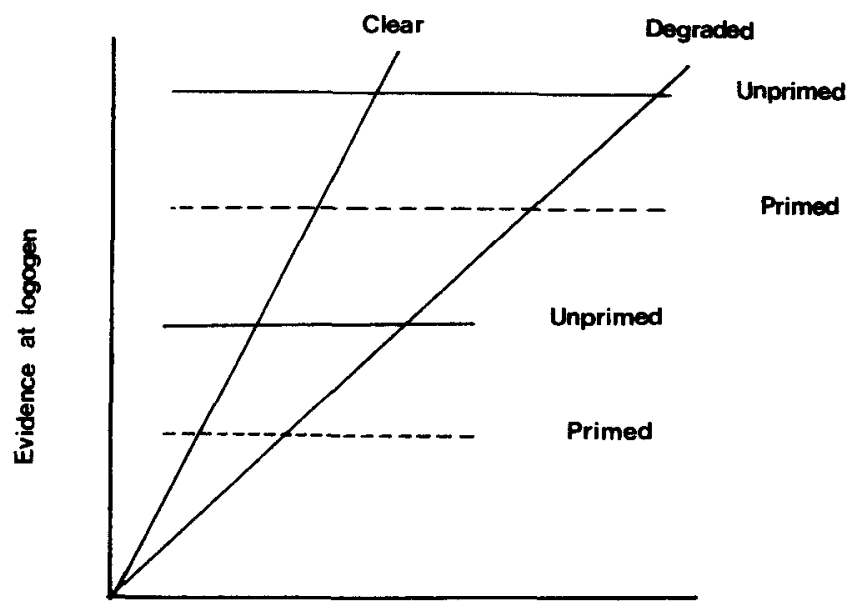

time since word onset

Figure 5. Morton's logogens are counting devices with preset thresholds. One possible representation is given above. Features pointing to a word having been presented can be assumed to be accumulated more rapidly under clear conditions, since featural information from the visual display can be extracted more readily. The upper pair of horizontal lines represent the highlevel thresholds for an item when it is "primed" or "unprimed." After a threshold level of featural information has been accumulated, the word corresponding to the logogen becomes available at a response buffer (Morton, 1970). The lower pair of horizontal lines represents a low-level threshold: After this level of information has been accumulated, access to the semantic system becomes possible from the particular logogen in question. From the particular representation given here, it is clear that priming and degradation interact. If the difference between the primed and unprimed levels are the same for both thresholds, then the interaction of priming and degradation should be constant. 
amount of sensory information required to produce a response, and he does not suggest that the extent of the reduction would depend on whether the high- or lowlevel criterion was being used. Accordingly, the lower pair of lines in Figure 5 are spaced the same as the upper pair. It should be clear from the geometry of this that the magnitude of priming effects and the magnitude of the interaction should be independent of which criterion is relevant to the task. In this way, the two-criterion logogen system is incapable of handling the differences between the two tasks.

There is one possible way in which a common encoding stage with two criterion levels could be maintained. If it could be assumed that priming effects were less for the semantic threshold than for the phonological threshold, then the interactions of degradation with $C F$ and relatedness would be reduced accordingly. Apart from the arbitrary nature of this assumption, a major difficulty is that there was no evidence whatsoever for an interaction between noise and CFE in the classification task, although there was a slight effect in the right direction for relatedness on the negative trials. The demonstration of additivity is always difficult, but if the present results were taken as providing such a demonstration, it would be necessary to assume total independence between the two tasks, and the concept of a common encoding stage would become meaningless.

There is evidence from several sources to indicate that the ability to name a word is not always a necessary concomitant of the word's activating semantic memory. The classic illustration of this is to be found in Marshall and Newcombe's (1966) work on an aphasic patient who could not always read stimulus words, but, rather, articulated semantically related words. The possibility that this is a process which could operate in normal subjects has been emphasized by Allport (in press), who showed that letter-masked word stimuli presented in a tachistoscope produced a number of errors which were reports of words semantically related to the actual target. Such demonstrations as these show the obscurity of the relationship between the ability to report a word and the word's influencing semantic memory. The present pair of experiments illustrate the obscurity once more, but this time with normal subjects and in relatively error-free reading conditions.

\section{REFERENCE NOTE}

1. Sanford, A. J., \& Garrod, S. Processing class membership information directly and indirectly. Paper presented to a meeting of the Experimental Psychology Society, Cambridge, July 1975.

\section{REFERENCES}

Allport, D. A. On knowing the meaning of words we are unable to report: The effects of visual masking. In $S$. Dornic (Ed.). Attention and performance $V I$, in press.

Battig, W. F., \& Montague, W. E. Category norms for verbal items in 56 categories: A replication and extension of the Connecticut category norms. Journal of Experimental Psychology Monograph, 1969, 80, 1-46.

Clark, H. H. The language-as-a-fixed-effect fallacy: A critique of language statistics in psychological research. Journal of Verbal Learning and Verbal Behavior, 1973, 12 , 335-359.

Collins, A. M., \& Quilian, M. R. Experiments on semantic memory and language comprehension. In L. W. Gregg (Ed.) Cognition in learning and memory. New York: Wiley, 1972.

JACOBSON, J. Z. Effects of association upon masking and reading latency. Canadian Journal of Psychology, 1973, 27, 58-69.

KIRK, R. E. Experimental design: Procedures for the behavioral sciences. Belmont, Calif: Brookes-Cole, 1968.

Landauer, T. K., \& Meyer, D. E. Category size and semantic memory retreival. Journal of Verbal Learning and Verbal Behavior, 1972, 11, 539-549.

Marshale, J. C., \& Newcombe, F. Syntactic and semantic errors in paralexia. Neuropsychologia, 1966, 4, 169-176.

Meyer, D. E., Schvaneveldt, R. W., \& Ruddy, M. G. Loci of contextual effects on visual word-recognition. In P. M. A. Rabbitt \& S. Dornic (Eds.) Attention and performance V. New York: Academic Press, 1975.

Morton, J. The effects of context on the visual duration thresholds for words. British Journal of Psychology, 1964, 55, 165-180.

Morton, J. A functional model for human memory. In D. A. Norman (Ed.) Models for human memory. New York: Academic Press, 1970.

Rips, L. J., Shoben, E. J., \& SMrth, E. E. Semantic distance and the verification of semantic relations. Journal of Verbal Learning and Verbal Behavior, 1973, 12, 1-20.

Rosch, E. On the internal structure of perceptual and semantic categories. In T. E. Moore (Ed.) Cognitive development and the acquisition of language. New York: Academic Press, 1973.

SAnford, A. J., \& SEYmour, P. H. K. The influence of response compatibility on a semantic classification task. Acta Psychologia, 1974, 38, 405-412.

SchaEffer, B., \& Wallace, R. The comparison of word meanings. Joumal of Experimental Psychology, 1970, 86, 144-152.

Smith, E. E., Shoben, E. J., \& Rips, L. J. Comparison processes in semantic memory. Psychological Review, 1974, 81, 214-241.

WILkINs, A. J. Conjoint frequency, category size and categorization time. Journal of Verbal Learning and Verbal Behavior, 1971, 10, 382-385.

\section{NOTES}

1. Standard errors (SEs) are derived from within-subjects variance.

2. Power calculations are based on standard errors for the by-subjects analysis only. Since this is more likely to detect an interaction than the min $F^{\prime}$ statistics, it is most appropriate to the argument. The results are virtually unaffected if the by-materials analysis is used, since the SEs are similar.

3. Context can also be thought of as setting the origin of the information accumulation function at a value above zero. This does not affect the argument and provides a rationale for the assumption that priming effects are equal for the two thresholds.

(Received for publication July 23, 1976; revision received October $20,1976$. 